\title{
Recurrent Laryngeal Nerve Palsy in Sarcoidosis Diagnosis and Management
}

\author{
Mohamed El-Amin*, Shahed Abdelmahmoud, Rajeev Advani and Khalid Ali \\ ${ }^{1}$ Core Surgical Trainee, North West Deanery, Manchester, UK \\ ${ }^{2}$ ENT Research Fellow, ENT department, The James Cook University Hospital, Middlesbrough, UK \\ ${ }^{3}$ ENT registrar, Northwest Deanery, Manchester, Uk \\ ${ }^{4}$ Consultant ENT surgeon, North West hospitals, NHS, UK
}

*Corresponding author: Mohamed El-Amin, core surgical trainee, Northwest

Deanery, Manchester, UK.

Received Date: January 19, 2021

Published Date: February 09, 2021

\section{Abstract}

Objectives: Vocal cord palsy secondary to sarcoidosis is a rare manifestation and it is very unusual for the patient to present with it as a chief complaint. This is the first literature review conducted highlighting the importance of this condition. 46 years lady presented with hoarseness of voice without any significant history related to upper airway or lung condition. Suffers only from obesity. Naso-endoscopic examinations showed left vocal cord palsy. CT scan of chest confirmed hilar lymphadenopathy which was diagnosed as sarcoidosis with endobronchial ultrasound (EBUS) guided lymph node biopsy. Symptoms were related to neuro-sarcoidosis and peripheral recurrent laryngeal nerve neuritis. No local compressive sings noted on imaging. The patient was treated with local vocal cord injection and steroids were not suggested considering the patient's obesity.

Methods: The literature review revealed only twenty-three reported cases. Number of cases included were 16 in relation to the nerve pathway. Cranial cases were excluded. One case report was due to peripheral neuro-sarcoidosis.

Results: The common practice was to manage with oral steroids, however, only few cases used injection laryngoplasty as treatment. Injection laryngoplasty is actively practiced in the UK under the National Institute of health and care Excellence (NICE) guidelines [ IPG130] which was published on the 22nd of June 2005.

Conclusion: Knowledge about vocal fold paralysis in sarcoidosis needs highlighting and should be considered by clinicians in cases that may initially appear to be idiopathic in nature without any chest complains related.

Keywords: Sarcoidosis; Recurrent laryngeal nerve; Paralysis; Palsy; Vocal cord injection; Mononeuritis multiplex; Dysphonia; Hoarseness of voice

\section{Background}

Hoarseness of voice secondary to recurrent laryngeal nerve palsy with mediastinal lymphadenopathy is usually thought of as malignancy, tuberculosis, pneumoconiosis, or a postoperative complication to the nerve [1,2]. Sarcoidosis has been implicated in few cases and this is usually managed by corticosteroids with good results [3]. The indications for vocal cord injection therapy have been expanded to include treatment for vocal cord paralysis, paresis, atrophy, and scar or sulcus [4]. Improvement in endoscopic techniques, injectable materials, and approach to injection has made utilizing this modality increasingly popular, though complex, for selected patients $[5,6]$. We describe below a patient who presented with hoarseness, proved to be secondary to sarcoidosis, treated with cord injection because of obesity. Also, we discuss the current literature evidence and the current practice in managing such conditions.

\section{Case Presentation}

A 46-year-old woman was referred to the otolaryngology department because of intermittent episodes of hoarseness of her voice for a period of six month. Her voice was squeaky at times but no other concomitant symptoms of weight loss, difficulty in breathing or in swallowing. She had a past medical history of carcinoma in situ of vulva which has cleared. She suffered from arthritis and 
hypertension. She is a non-smoker with history of minimal alcohol intake. A good history and clinical examination were carried out searching for potential red flag of the above mentioned (referred otalgia, haemoptysis, dysphagia, odynophagia, neck lump and smoking history) and there was nothing of the sort in our case. Clinical examination was unremarkable, in particular her chest was clear and there were no palpable lymph nodes in her neck. Flexible nasolaryngoscopy did not show any obvious mucosal lesion in her upper aero-digestive tract but noted palsy of her left vocal cord. For that reason, urgent CT neck and thorax were ordered.

CT scan showed a larynx with features of left vocal cord palsy or weakness. The left laryngeal ventricle was asymmetrically enlarged, and the arytenoid cartilage was deviated medially. However, no laryngeal masses were seen. Positron emission tomography (PET) imaging showed no signs of neck lymphadenopathy. The thyroid, submandibular and parotid glands were all normal anatomy. In the thorax there were enlarged mediastinal lymph nodes in the right paratracheal, perivascular, subcarinal and both bilateral hilar locations. The subcarinal lymph node measured $22 \mathrm{~mm}$ x $12 \mathrm{~mm}$. The perivascular measured $25 \mathrm{~mm} \times 16 \mathrm{~mm}$. The right paratracheal lymph node was partly obscured by streak artefact but still looked enlarged. No signs of any obvious lesions involving the bronchial tree. No evidence of lung parenchymal disease was noted or masses. After contrast, the mediastinal lymph nodes enhanced but there was no enhancement of the axillary lymph nodes or any sinister areas involving the musculoskeletal system.

On the light of the above findings the differential diagnosis of sarcoid, lymphoma and infections were entertained, and an urgent referral was made to the respiratory physician. Patient was then sent for hilar biopsies guided by endobronchial ultrasound (EBUS). Biopsy of the subcarinal node confirmed granulomatous disease consistent with the diagnosis of sarcoidosis. It was decided not to put the patient on steroids as she got a high BMI at 34. Also, as she did not complain of any chest symptoms, the respiratory physicians were happy not to put her on any active management measures. Left vocal cord medialization with cord injection using Radiesse was indicated to help improving her symptoms. Patient was reviewed after the procedure and naso-endoscopy showed closure of the phonation gab. As patient showed good progress, it was planned to be followed up in clinic for further assessment if she needs any more vocal cord injection in future.

\section{Discussion}

Dysphonia is a common presenting symptom to the otolaryngology outpatient department. Although acute laryngitis is the most common transient cause of dysphonia, there are arrays of pathologies that can give rise to this symptom. Hoarseness can result from a wide spectrum of conditions ranging from the common cold to a malignancy. Because of its prevalence and the multitude of disease processes that can be accountable, it can be present a real diagnostic challenge. Although, the term hoarseness is suitable as a chief complaint, it is vague and nonspecific. However, for normal voice production we need optimal posturing of the 2 vocal folds in contact or near contact; and proper internal tension of the vocal folds to produce the desired pitch and voice quality [7].

If normal voice is a series of pressure waves with a certain shape produced by normal vocal folds, then an aberration on the vibrating edge of a vocal fold, a change in the distance between the 2 vibrating edges, or a change in the internal tension of the vocal folds can all change the shape of the pressure waves, which are then perceived as an altered sound or hoarseness. Each of these elements needs to be considered to reach solution to the problem of hoarseness [7]. The differential diagnosis of hoarseness should start with the common conditions like laryngitis, muscle tension dysphonia, laryngopharyngeal reflux (LPR), allergy or benign vocal cord lesions like unilateral benign vocal cord lesions. Vocal polyp, granuloma, papilloma, and vocal cord nodules [811]. Other conditions are associated with mediastinal lesions like tuberculosis, sarcoidosis, or pneumoconiosis. Systemic diseases are often incriminated like hypothyroidism, amyloidosis, rheumatoid arthritis, dystonia a functional vocal cord paresis [12].

Laryngeal malignancy that manifests as hoarseness is a mustnot-miss diagnosis. It is not possible to distinguish malignant lesions from benign lesions based on history and auditory perception alone, although a history of smoking should greatly increase the clinician's suspicion for a malignant process. Of all the possible causes of hoarseness, it is this entity that makes laryngoscopy indispensable in the assessment of hoarseness that does not resolve in a short amount of time. The most common laryngeal malignancy is squamous cell carcinoma (SCC) [13]. With approximately $56 \%$ originating on the vocal folds and therefore would manifest first as hoarseness. Because the survival and treatment morbidity of laryngeal SCC correlate with the extent of the disease, timely diagnosis and treatment is of paramount importance [14].

\section{Literature Review}

Literature search was conducted via the NHS ATHENS evidence website, and an advanced healthcare database search was conducted (Figure 1): Showing the search included the following databases: MEDLINE, PubMed and EMBASE and other grey sources of data (Figure 1) (Table 1, 2).

Table 1: Summary of Reported Cases of Sarcoidosis-associated Vocal Fold immobility and has been included in our study.

\begin{tabular}{|c|c|c|c|c|c|}
\hline Case & Age & Site & Treatment & Cause & Author \\
\hline 1 & $49 \mathrm{~F}$ & Bilateral & Tracheostomy, Radiesse injection, and oral steroids & Direct invasion & Hintze JM 2018 \\
\hline 2 & $72 \mathrm{~F}$ & Bilateral & Oral steroids $(4 \mathrm{mo})$ & Lymphadenopathy & Yamasue M 2016 \\
\hline 3 & $40 \mathrm{~F}$ & Left & PEG) $(6 \mathrm{wk})$ & Lymphadenopathy & Mastan S 2015 \\
\hline
\end{tabular}




\begin{tabular}{|c|c|c|c|c|c|}
\hline 4 & $61 \mathrm{M}$ & Bilateral & PEG) (3 mo) & Lymphadenopathy & Lop Gros J 2014 \\
\hline 5 & $35 \mathrm{~F}$ & Left & Oral steroids (6 mo) & Lymphadenopathy & Lop Gros J 2014 \\
\hline 6 & $60 \mathrm{~F}$ & Left & Oral steroids (2 y) & Lymphadenopathy & Sekiguchi H 2013 \\
\hline 7 & $53 \mathrm{M}$ & NA & Oral steroid & Direct laryngeal involvement & Alon EE 2010 \\
\hline 8 & $48 \mathrm{M}$ & Right & NA & Neuritis & Alon EE 2010 \\
\hline 9 & $45 \mathrm{M}$ & Right & Oral steroids (2 mo) and lymphadenectomy & Lymphadenopathy & Boyd M 2011 \\
\hline 10 & $35 \mathrm{M}$ & Bilateral & IV steroids (3 d) and oral steroids (indefinitely) & Lymphadenopathy & Coffey CS 2009 \\
\hline 11 & $74 \mathrm{~F}$ & Left & NA & Lymphadenopathy & Hughes P 1995 \\
\hline 12 & $64 \mathrm{M}$ & Left & Oral steroids (8 mo) & Lymphadenopathy & Tobias JK 1990 \\
\hline 13 & $49 \mathrm{M}$ & Left & Oral steroids & Lymphadenopathy & el-Kassimi 1990 \\
\hline 14 & $45 \mathrm{~F}$ & Left & Oral steroids & Lymphadenopathy & Swinburn 1986 \\
\hline 15 & $25 \mathrm{M}$ & Left & Spontaneous resolution after (10 mo) & Lymphadenopathy & Chijimatsu Y 1980 \\
\hline 16 & $17 \mathrm{M}$ & Left & Oral steroids &
\end{tabular}

Abbreviations: F: Female; IV: Intravenous; L: Left; M: Male; NA: Not Available.

Table 2: Summary of Reported Cases of Sarcoidosis-associated Vocal Fold immobility and has been excluded from our study.

\begin{tabular}{|c|c|c|c|c|c|}
\hline Case & Age & Site & Treatment & Cause & Author \\
\hline 1 & $57 \mathrm{~F}$ & Bilateral & NA & Cranial polyneuritis & Crossley JR \\
2020
\end{tabular}

Abbreviations: F: Female; IV: Intravenous; L: Left; M: Male; NA: Not Available.

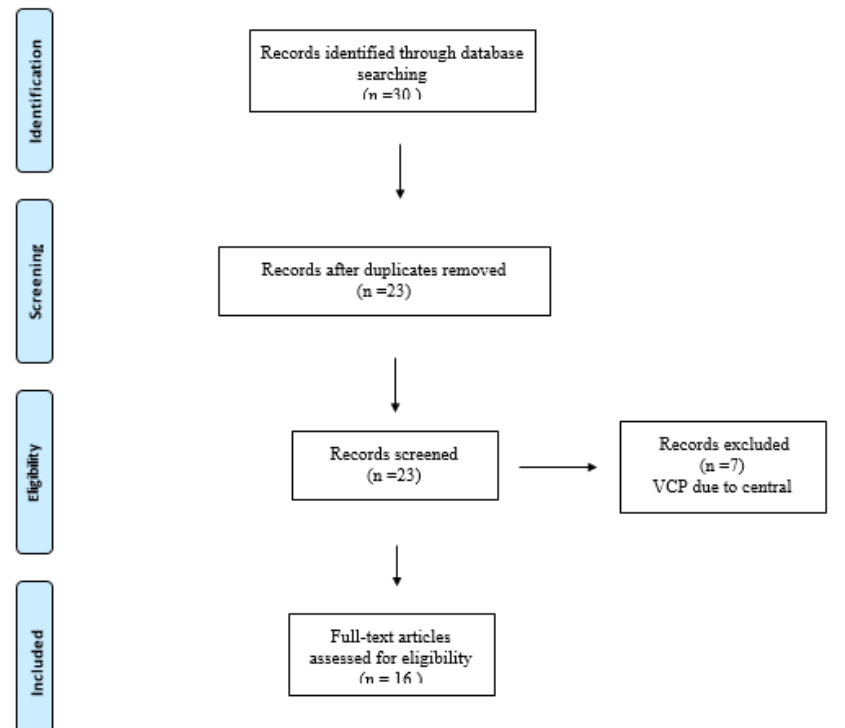

Figure 1: Flow diagram of the literature search. PubMed, Embase, Medline databases and other internet sources were used to identify records. Screening records included reading abstracts. Full text articles for final analysis were selected if they included VCP due peripheral causes in their description. 
A search of the literature revealed twenty-three reports of vocal cord paralysis discovered in patients who were subsequently diagnosed with sarcoidosis. (Table 1): showing the included studies showing the related cause to peripheral neuritis. (Table 2): showing the cases in relation to central causes with brain and spinal cord involved. The average age of presentation was between 40-50 years. Men were involved in 13 out of the 23 cases (56\%), and the left vocal fold was most frequently affected (47\%), likely because of the unique intrathoracic course of the left recurrent laryngeal nerve as it passes around the arch of the aorta and it has a distance of $12 \mathrm{~cm}$, compared with $6 \mathrm{~cm}$ for the right recurrent laryngeal nerve. Owing to its longer course, it is more susceptible to damage compared with the right recurrent laryngeal nerve. Two cases of right-sided vocal fold paralysis have been reported [3, 15]. Bilateral vocal fold immobility has been reported in eight cases, four of them were due to central cause [16-18].

Reports of unilateral left vocal cord palsy are more common probably due to compression by the bulky mediastinal nodes on the left recurrent laryngeal nerve at the level of the arch of the aorta. Mechanisms of nerve palsy not fully understood till today, however the common etiology found in literature is compressive mediastinal lymphadenopathy, direct laryngeal involvement and cranial polyneuritis $[16,17]$. Most of the theories come around local compression mechanisms. However, recurrent laryngeal nerve neuritis had been mentions as part of the inflammatory process of sarcoidosis. Confirmation of that would be only possible in post mortal examination through tissue biopsies. Form our literature, it appears this is the second case resulted as consequence of mononeuritis associated with sarcoidosis leading to recurrent laryngeal nerve palsy, in absences of midsternal mass pressure or apparent vocal cord lesions on examinations [19,21].

The presenting symptom in each of these cases was hoarseness, and several patients were noted to have cough or dysphagia. In each case, a chest radiograph or chest CT scan demonstrated significant enlargement of hilar, peritracheal, or mediastinal lymph nodes, which were believed to be the cause of vocal cord paralysis. The problem aroused when it was recognised that the patient was obese with a BMI of 34 which contradicted giving her steroids for treatment particularly that she was asymptomatic concerning her sarcoidosis. Therefore, we opted for vocal cord injection to relief her hoarseness. In the broadest sense, vocal cord injection can include procedures that target the superficial (sub-epithelial space) aspect of the vocal fold. This procedure involves injection of a substance as a lamina propria replacement. Useful for mild-to-moderate vocal fold scar and lamina propria defects, superficial injection provides correction of vibratory defects rather than global augmentation. This patient was injected with good short-term effects. She might need repeat injections if her symptoms recur.

The (Laryngeal Electromyography) LEMG could have strengthened the diagnosis and it is also helpful in the follow up of the patient $[21,22]$ but our patient presented late after onset of symptoms and also EMG was not readily available to us. In this patient the Voice Handicap Index (VHI) was not done but she made impressive improvement following the intervention evident by patient satisfaction with results. (VHI) was developed by Jacobson in 1998s and is currently one of the most widely used instruments for the evaluation of patient-perceived vocal incapacity. It is a selfreport questionnaire divided into three subscales (functional, organic, and emotional) each with 10 questions [22]. These questions were originally selected on the basis of the analysis of case reports, to ensure that the scale has nominal content and validity. The functional subscale explores the effects of the voice disorder on daily activity; the organic subscale explores the patient's perception of the characteristics of his/her vocal production, and of laryngeal discomfort; the emotional subscale explores the patient's effective responses to the problem. The test is applicable to all types of vocal disorders and has been statistically validated [22]

\section{Conclusion}

A 46-year-old female presented to the ENT department with only hoarseness of voice, her physical examination was normal but nasolaryngoscopy showed immobility of her left vocal cord and imaging revealed a silent mediastinal mass. Biopsy confirmed sarcoidosis. Because of her obesity she did not receive corticosteroids. She had vocal cord injection that helped relieving her hoarseness. Therefore, otolaryngologists should be aware of the rare occurrence and presentation of VCP in sarcoidosis, as prompt diagnosis by brain MRI, as opposed to traditional chest radiography or computed tomography, followed by serial laryngeal examinations is necessary for management and treatment. Surgical treatment with injection or medicalization laryngoplasty can improve voice quality. There were cases reported utilizing EMG to help determine the true pathophysiology and elucidating the cause of immobility. If laryngeal EMG is suggestive of neural injury, neural biopsy may further help to differentiate between direct neural invasion and neurosarcoidosis but that only possible in autopsy. A normal EMG will be a strong suggestion for direct infiltration of the larynx rather than neural involvement [23]. The serum angiotension-converting enzyme (ACE) levels not been utilized as it lacks the sufficient specificity as a diagenetic tool [24-36].

\section{Acknowledgement}

None.

\section{Conflict of Interest}

No conflict of interest to declare about this publication.

\section{References}

1. Hamilton JRL, Varghese G, Shepperd HWH, Stevenson HM (1986) Tuberculous left vocal cord palsy: a timely reminder. J Laryngol Otol 100: 837-838.

2. Sherani TM, Angelini GD, Passani SP, Butchart EG (1984) Vocal cord paralysis associated with coalworkers' pneumocon-iosis and progressive massive fibrosis. Thorax 39: 683-684. 
3. Boyd M, Malalsamy S, Le S (2011) Right vocal cord paralysis and mediastinal lymphadenopathy. Thorax 66: 211-231.

4. Laura H Swibel Rosenthal, Michael S Benninger, Robert H Deeb (2007) Vocal Fold Immobility: A Longitudinal Analysis of Etiology Over 20 Years. The Laryngoscope 117(10): 1864-1870.

5. Rosen CA, Amin MR, Sulica L, Simpson CB, Merati AL, et al. (2009) Advances in office-based diagnosis and treatment in laryngology. Laryngoscope 119(Suppl 2): S185-S212.

6. Coffey CS, Vallejo SL, Farrar EK (2009) Sarcoidosis presenting as bilateral vocal cord paralysis from bilateral compression of the recurrent laryngeal nerves from thoracic adenopathy. J Voice 23: 631-634.

7. Ted Mau (2010) Diagnostic Evaluation and Management of Hoarseness. Med Clin N Am 94: 945-960.

8. Cohen SM, Garrett CG (2008) Hoarseness: is it really laryngopharyngeal reflux? Laryngoscope 118: 363-366.

9. Garrett CG, Cohen SM (2008) Otolaryngological perspective on patients with throat symptoms and laryngeal irritation. Curr Gastroenterol Rep 10: 195.

10. Cohn JR, Spiegel JR, Sataloff RT (1995) Vocal disorders and the professional voice user: the allergist's role. Ann Allergy Asthma Immunol 74: 363-366

11. Dworkin JP, Reidy PM, Stachler RJ (2009) Effects of sequential Dermatophagoides pteronyssinus antigen stimulation on anatomy and physiology of the larynx. Ear Nose Throat J 88: 793-799.

12. Altman KW, Atkinson C, Lazarus C (2005) Current and emerging concepts in muscle tension dysphonia: a 30-month review. J Voice 19: 261-267.

13. National Cancer Institute website. Surveillance epidemiology and end results.

14. Shah JP, Karnell LH, Hoffman HT (1997) Patterns of care for cancer of the larynx in the United States. Arch Otolaryngol Head Neck Surg 123 475-483.

15. Alon EE, Ekbom DC (2010) Neurosarcoidosis affecting the vagus nerve. Ann Otol Rhinol Laryngol. 119: 641-645.

16. Crossley JR, Aminpour N, Giurintano JP, Jay AK, Harris BT, et al. (2021) Neurosarcoidosis Directly Involving the Cervical Vagus Nerve. Ann Otol Rhinol Laryngol 130(2): 215-218.

17. Witt RL (2003) Sarcoidosis presenting as bilateral vocal fold paralysis. J Voice17(2): 265-268.

18. Wu TJ, Lewis SM, Woo P (2019) Neurosarcoidosis presenting initially as idiopathic vocal cord paralysis. Ann Otol Rhinol Laryngol 128(2): 157161.

19. Saleem Mastan, Rajeev Advani, Nicola Stobbs, Nirmal Kumar (2015) A rare manifestation of a multisystemic disese: a case of vocal cord palsy secondary to sarcoidosis. BMJ Case Rep.
20. Jaime I Chang, Scott E Bevans, Seth R Schwartz (2012) The role of EMG in diagnosis of laryngeal sarcoidosis. Otolaryngology Clinic of North America 45(5): 1109-1126.

21. Chen-Chi Wang, Ming-Hong Chang, Armando De Virgilio (2014) Laryngeal Electromyography and Prognosis of Unilateral Vocal Fold Paralysis-A Long-term Prospective Study. Laryngoscope 898-903.

22. BH Jacobson, A Johnson, C Grywasky (1998) The Voice Handicap Index (VHI): development and validation. J Voice 12: 540-550.

23. Hintze JM, Gnagi SH, Lott DG (2018) Sarcoidosis presenting as bilateral vocal fold immobility. J Voice 32(3): 359-362.

24. Studdy PR, Bird R (1989) Serum angiotensin converting enzyme in sarcoidosis-its value in present clinical practice. Ann Clin Biochem 26(pt1): 13-18.

25. Rosen CA, Murry T (2000) Nomenclature of voice disorders and vocal pathology. Otolaryngol Clin North Am 33: 1035-1046.

26. Agrawal R, Inota JA, Akin EA (2013) Prevalence of vocal cord paralysis in patients with incidentally discovered enlarged lymph nodes along the expected course of the recurrent laryngeal nerve. Ann Otol Rhinol Laryngol 122: 229-234.

27. Jaffe R, Bogomolski Yahalom V, Kramer MR (1994) Vocal cord paralysis as the presenting symptom of sarcoidosis. Respir Med 88: 633-636.

28. El-Kassimi, Ashour, Vijayaraghavan (1990) Sarcoidosis presenting as recurrent left laryngeal nerve palsy. Thorax 45(7): 565-566.

29. Yamasue M, Nureki S, Ushijima R, Mukai Y, Goto A et al. (2016) Sarcoidosis Presenting as Bilateral Vocal Cord Paralysis due to Bilateral Vagal Nerve Involvement. Intern Med 55: 1229-1233.

30. Mastan S, Advani R, Stobbs N, Kumar N (2015) A rare manifestation of a multisystemic disease: a case of vocal cord palsy secondary to sarcoidosis. BMJ Case Rep.

31. Lop Gros J, Garcia Lorenzo J, Quer Agusti M (2014) Sarcoidosis and true vocal fold paresis: 2 cases and a review of the literature. Acta Otorrinolaringol Esp 65: 378-380.

32. Hughes P, McGavin C (1995) Recurrent laryngeal palsy and mediastinal lymphadenopathy. Respir Med 89: 584-585.

33. Jaffe R, Bogomolski-Yahalom V, Kramer MR (1994) Vocal cord paralysis as the presenting symptom of sarcoidosis. Respir Med 88: 633-636.

34. Tobias JK, Santiago SM, Williams AJ (1990) Sarcoidosis as a cause of left recurrent laryngeal nerve palsy. Arch Otolaryngol Head Neck Surg 116: 971-972.

35. Swinburn CR, Pozniak AL, Davies DG, Treasure T, Johnson NM (1986) Left recurrent laryngeal nerve palsy as the presenting feature of sarcoidosis. 3(1): 67-68.

36. Chijimatsu Y, Tajima J, Washizaki M (1980) Hoarseness as an initial manifestation of sarcoidosis. Chest 78: 779-781. 